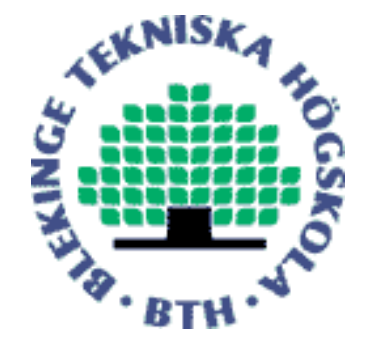

Copyright (C) 2014 IEEE.

Citation for the published paper:

A PMIPv6 Approach to Maintain Network Connectivity During VM Live Migration Over the Internet

Solomon Kassahun, Atinkut Demessie, Dragos Ilie

International Conference on Cloud Networking (CloudNet)

2014 Luxembourg

This material is posted here with permission of the IEEE. Such permission of the IEEE does not in any way imply IEEE endorsement of any of BTH's products or services Internal or personal use of this material is permitted. However, permission to reprint/republish this material for advertising or promotional purposes or for creating new collective works for resale or redistribution must be obtained from the IEEE by sending a blank email message to pubs-permissions@iee.org.

By choosing to view this document, you agree to all provisions of the copyright laws protecting it. 


\section{A PMIPv6 Approach to Maintain Network Connectivity During VM Live Migration Over the Internet}

\author{
Solomon Kassahun \\ Blekinge Institute of Technology \\ Dept. of Comm. Systems \\ Karlskrona, Sweden \\ E-mail: sokc11@ student.bth.se
}

\author{
Atinkut Demessie \\ Blekinge Institute of Technology \\ Dept. of Comm. Systems \\ Karlskrona, Sweden \\ E-mail: atde11@student.bth.se
}

\author{
Dragos Ilie \\ Blekinge Institute of Technology \\ Dept. of Comm. Systems \\ Karlskrona, Sweden \\ E-mail: dragos.ilie@bth.se
}

\begin{abstract}
We present a live migration solution based on Proxy Mobile IPv6 (PMIPv6), a light-weight mobility protocol standardized by IETF. PMIPv6 handles node mobility without requiring any support from the moving nodes. In addition, PMIPv6 works with IPv4, IPv6 and dual-stack nodes. Our results from a real testbed show that network connectivity is successfully maintained with little signaling overhead and with short virtual machine (VM) downtime. As far as we know, this is the first time PMIPv6 is used to enable live migration beyond the scope of a LAN.
\end{abstract} nodes

Keywords-Protocols, computer network management, mobile

\section{INTRODUCTION}

Current virtualization packages use simple techniques to preserve network connectivity during live migration over a local area network (LAN). They broadcast a gratuitous Address Resolution Protocol (ARP) message, in the case of IPv4, or multicast an unsolicited Neighbor Advertisement (NA) message in the case of IPv6. The message forces the LAN nodes to update their ARP table, or neighbor caches in the case of IPv6, so that the IP address of the VM becomes associated with the link-layer address of the new host [1]. However, this approach cannot be applied beyond a broadcast domain.

Being able to move VMs between geographically separated data centers can be useful in several situations [2]. For example, cloud bursting is a technique where an organization temporarily migrates its servers to the cloud to handle short periods of intensive load. When the load returns to more acceptable levels, the servers are migrated back to the original hosts. Another case for live migration over a wide area network (WAN) is when enterprises consolidate services from smaller sites into one or several larger data centers. In this scenario, large application downtimes are undesirable and live migration provides the means to meet this demand. Finally, companies with offices in different time zones may choose to implement a "follow the sun" strategy to offer services and keep projects active around the clock. Using this strategy, teams working during business hours can pick up work from a team in a different time zone where the business hours have ended. In this situation, live migration can be a useful alternative to replicating data among geographically separated sites.

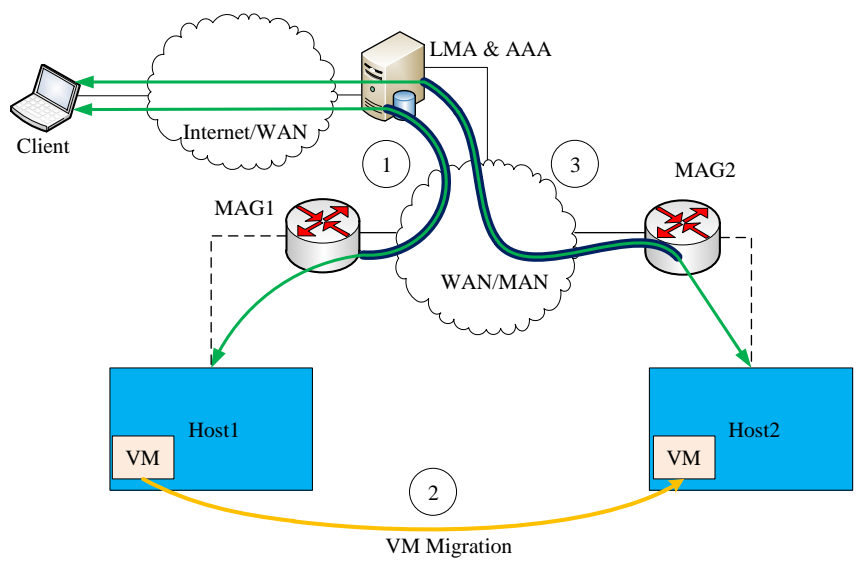

Fig. 1. PMIPv6 approach

We have implemented a solution based on Proxy Mobile IPv6 (PMIPv6), which is a light-weight mobility protocol standardized by IETF. PMIPv6 [3] handles node mobility inside an administrative domain without requiring any support from the moving nodes. In addition, PMIPv6 works with IPv4, IPv6 and dual-stack nodes [4]. Consequently, there is no need to enable any specific features in migrating VMs. Our results from a real testbed show that network connectivity is successfully maintained with little signaling overhead and with short VM downtime. As far as we know, this is the first time PMIPv6 is used to enable live migration beyond the scope of a LAN.

In addition, we have compared our solution to a similar approach [5] based on Mobile IPv6 (MIPv6). Our solution is able to provide a considerable shorter service downtime than the MIPv6 approach.

\section{Migration in A PMiPv6 Domain}

In our approach, PMIPv6 provides network-based mobility support to a migrating VM without involving it in mobility related signaling. The PMIPv6 entities, Mobile Access Gateway (MAG) and Local Mobility Anchor (LMA), keep track of the VM's movement, initiate the mobility signaling, and setup the required routing states. 


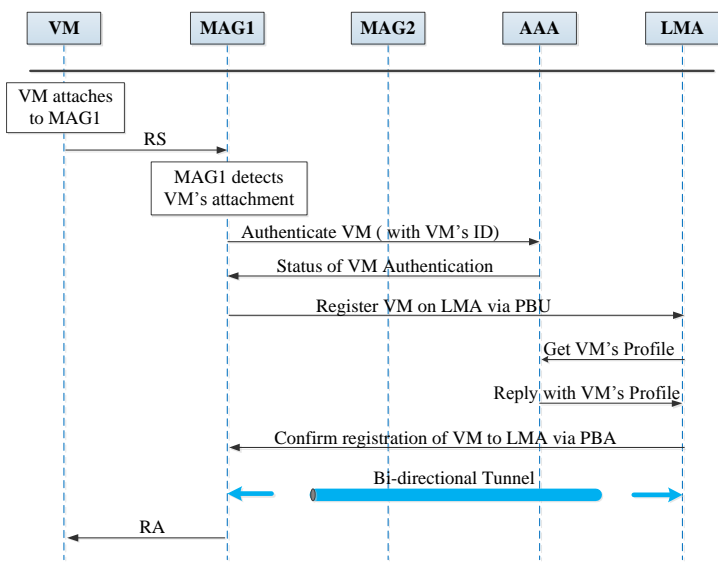

Fig. 2. VM entrance in the PMIPv6 domain

Fig. 1 illustrates the architecture of our testbed. Host 1 accommodates a virtual machine, VM. Clients, whether inside or outside the PMIPv6 domain, can access services provided by VM. The node Client is an example of such client. An Authentication Authorization Accounting (AAA) server is running on the same node as the LMA.

A bi-directional tunnel is established between MAGI and LMA after VM is booted up and authorized to enter the PMIPv6 domain. Initially, data packets exchanged between $\mathrm{Client}$ and VM traverse the tunnel established between LMA and MAG1. This event is shown as (1) in Fig. 1. At some point VM migrates from Host 1 to Host2. This event is marked by (2) in Fig. 1. After VM has been successfully migrated to Host2, a new tunnel will be formed between LMA and MAG2. The tunnel between LMA and MAG1 is then torn down in order to release resources reserved for it. Afterwards, data packets between Client and VM1 will travel through the new tunnel. This event is marked by (3) in Fig. 1. These events are explained below in more detail.

\section{A. VM Entrance in the PMIPv6 Domain}

Fig. 2 shows the sequence of events that occur when the VM node enters a PMIPv6 domain after it is booted up. We assume here that VM was turned off long enough for its Binding Cache entrys (BCEs) from a previous instantiation to be evicted from the binding cache in the LMA.

After VM is booted up it attaches to the access link and sends a Router Solicitation (RS) message to obtain valid network prefixes for autoconfiguration. In our testbed, VM and MAGI are hosted on the same Linux machine, and a virtual bridge is used to emulate the access link that both VM and MAG1 share, as shown in Fig. 4.

MAG1 uses the reception of the RS message as an indication that VM is attached to its link. This triggers MAG1 to begin the access authentication procedure with the AAA server. If $\mathrm{VM}$ is authorized to join the PMIPv6 domain, then MAG1 sends a Proxy Binding Update (PBU) message to establish a binding on the LMA. The source address of the message is set to the Proxy care-of address (CoA), which is the address of MAGI's egress interface.

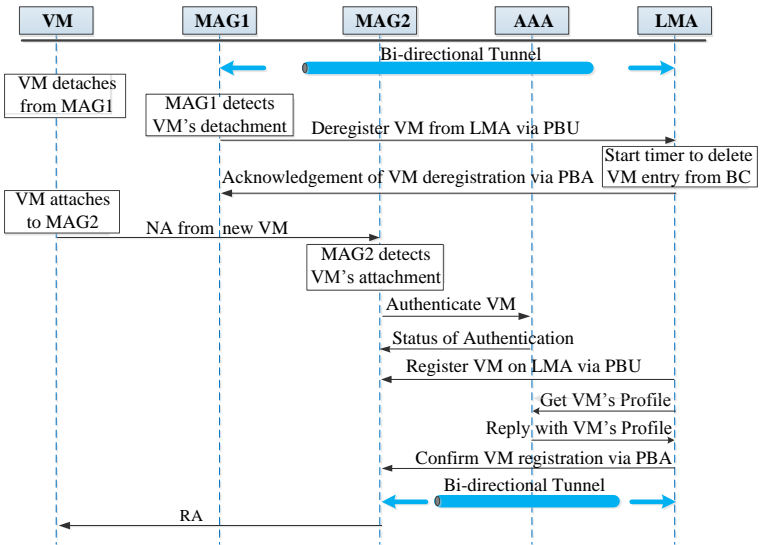

Fig. 3. VM migration in the PMIPv6 domain

When LMA receives the PBU message, it queries the AAA server to determine if MAGI is authorized to operate on behalf of VM and if VM is authorized for network-based mobility management service. If authorization succeeds, LMA proceeds with the next step, which is to check if VM has an active BCE. In this case, no entry is found because VM is just entering the PMIPv6 domain. Thus, a new entry is created for it. LMA allocates one or more home network prefixes (HNPs) to VM depending on the policies configured on the AAA server. Then, LMA establishes a bi-directional tunnel to MAGI and updates its routing tables so that packets going towards the allocated HNPs are sent through the tunnel.

LMA finishes the binding procedure by sending a Proxy Binding Acknowledgement (PBA) message to MAG1. The PBA message carries the HNPs assigned to VM. After receiving the PBA, MAGI updates its routing tables to enable forwarding for packets using the received HNPs. Finally, MAG1 sends a Router Advertisement (RA) message advertising the received HNPs to VM's link-local address. This allows VM to complete autoconfiguration.

\section{B. VM Migration within the PMIPv6 Domain}

Fig. 3 shows the sequence of events that occur when VM is migrated from the source host attached to MAG1 to the destination host attached to MAG2.

There are several ways to detect a node's detachment from a MAG, depending on the type of access link between the mobile node (MN) and the MAG [3]. We have used the Neighbor Unreachability Detection (NUD) event [6] for this purpose.

In fact, the attachment and detachment of VMs could be precisely detected by modifying the hypervisor to notify the MAG directly when these events occur. Although this would lead to faster movement detection, it would also make the solution complex and hypervisor dependent. Therefore, we chose to utilize the generic features of the hypervisor, which are common among several virtualization platforms.

After MAG1 detects MN's detachment, it sends a PBU message to LMA to unregister the current binding. MAGI 


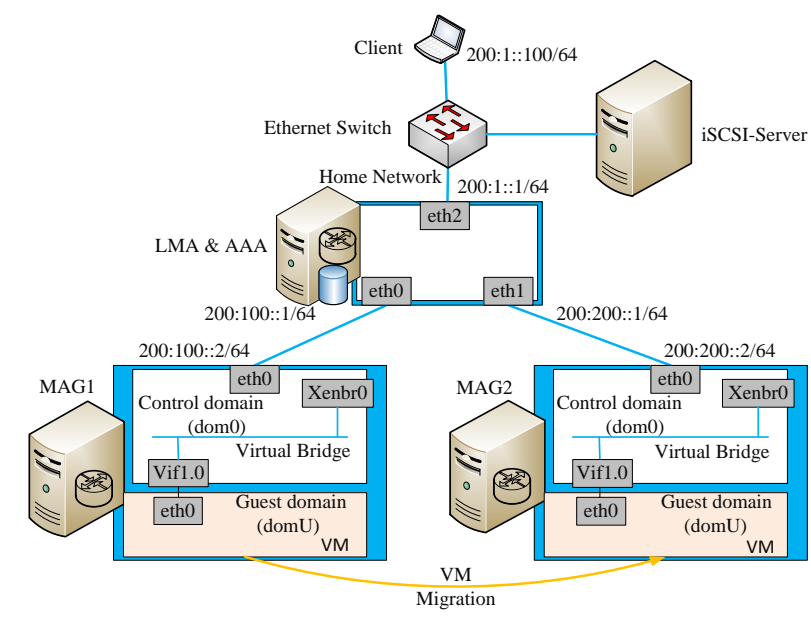

Fig. 4. Testbed architecture

deletes all state associated with $\mathrm{VM}$ after receiving a PBA reply or after an associated timer expires.

When receiving the PBU message from MAG1, LMA checks that a corresponding valid $\mathrm{BCE}$ exists in its cache. If found, LMA sends a PBA message to MAG1 to confirm the deregistration request. Then, LMA enters a waiting state and removes all routes associated with VM. However, it will not delete VM's BCE entry yet. The waiting time allows MAG2 to detect VM's attachment and to send a registration PBU message to LMA.

VM sends a unsolicited NA message after it resumes operation on the new host. MAG2 uses the message as an indication that $\mathrm{VM}$ is attached to its access link. This triggers $M A G 2$ to contact the AAA server for $V M$ authentication. If the authentication succeeds, MAG2 sends a PBU message to LMA to register the new binding. After receiving the message, LMA verifies with the AAA server if MAG2 is authorized to send the $\mathrm{PBU}$ message. If it is, LMA continues to process the message. Since the old BCE for VM is still available, LMA needs only to update the Proxy CoA field in the BCE with the address of MAG2's egress interface. Then, LMA updates its routing tables, establishes bi-directional tunnel to MAG2, and sends a PBA reply to MAG2.

When receiving the PBA reply, MAG2 updates its routing table and copies the VM's HNPs from the PBA message into a RA message that is sent over the access link. Because the HNPs did not change, VM's IP address(es) remain unchanged after $\mathrm{VM}$ receives the RA message.

Note that MAG2's registration may arrive to LMA before the de-registration message from MAG1. In this case, LMA removes any previous routes to MAG1 established on behalf of $\mathrm{VM}$ and then it processes the request as described above. When the de-registration message from MAGI arrives it is ignored.

\section{TESTBED}

As shown Fig. 4, the testbed consists of five machines all running the Ubuntu 12.04 LTS operating system (OS).
LMA acts as a central router in our testbed. A WAN is emulated by introducing latency on LMA's links using Linux's network emulator NetEm. LMA is also configured as a centralized AAA server. We used the FreeRADIUS server for Linux to implement AAA functionality. The AAA server hosts a central database that holds VM's profile, which includes VM's ID, password, and HNP. LMA, MAG1 and MAG2 are clients to the AAA server.

MAG1 and MAG2 serve dual roles as MAGs and VM hosts. They are directly connected to LMA's gigabit Ethernet interfaces. The access link that the MAGs and VM share is emulated using Linux bridge as provided by the bridge-utils package. In addition, FreeRADIUS client software obtained from [7] is installed on MAG1 and MAG2.

VM is a paravirtualized guest which runs Ubuntu 12.04 LTS OS with a kernel built from the linux-3.2.0-37-generic-pae source code.

$\mathrm{Client}$ represents an external user who accesses services running on VM. The iSCSI-Server node provides a shared storage medium to MAG1 and MAG2 using the iSCSI protocol. VM's disk state is saved on iSCSI-Server. Both MAG1 and MAG2 are configured as iSCISI clients. We installed the iscsitarget Linux package on the iSCSI-Server and the open-iSCSI package on the MAGs.

We installed the xen-hypervisor-amd64 package on MAG1 and MAG2 to obtain access to the control domain (dom0) in our testbed. Xen is enabled by default in the generic Ubuntu 12.04 LTS kernel.

\section{A. PMIPv6 Software Distribution}

We used EURECOM's [7] implementation of PMIPv6 with a small modification. The implementation relies on RS messages to indicate when a node attaches to a MAG. They are sent as part of the network configuration step, for example when a VM boots up. Unfortunately, a VM will not emit a RS message after live migration, because the move is transparent to the layer-3 network state. However, after migration the destination hypervisor either sends an unsolicited NA message on behalf of the VM, or "tricks" the guest OS into doing that itself. Therefore, we changed EURECOM's source code to allow MAGs to infer a VM's attachement from the reception of unsolicited NA messages sent by a node that is not already registered as attached to the MAG.

Testbed elements LMA, MAG1 and MAG2 run pmip6d, the PMIPv6 daemon. The daemon requires MAGs to use mobilityready kernels, similar to those used in evaluating the MIPv6 approach, as explained below.

\section{B. Testbed Adaptation for MIPv6 Approach}

The physical architecture of the testbed remains the same when using the testbed for MIPv6 approach described in [5]. However, in this case the LMA node serves as home agent (HA) while MAG1 and MAG2 act as access routers on foreign links. UMIP's implementation [8] of MIPv6 is installed on both LMA and VM. The router advertisement daemon radvd [9] runs on MAG1 and MAG2. The daemon on MAG1 and MAG2 is configured to send RA message at an interval of $0.07 \mathrm{~ms}$ as recommended in [10]. The iSCSI-Server and Client nodes retain their previous roles. 


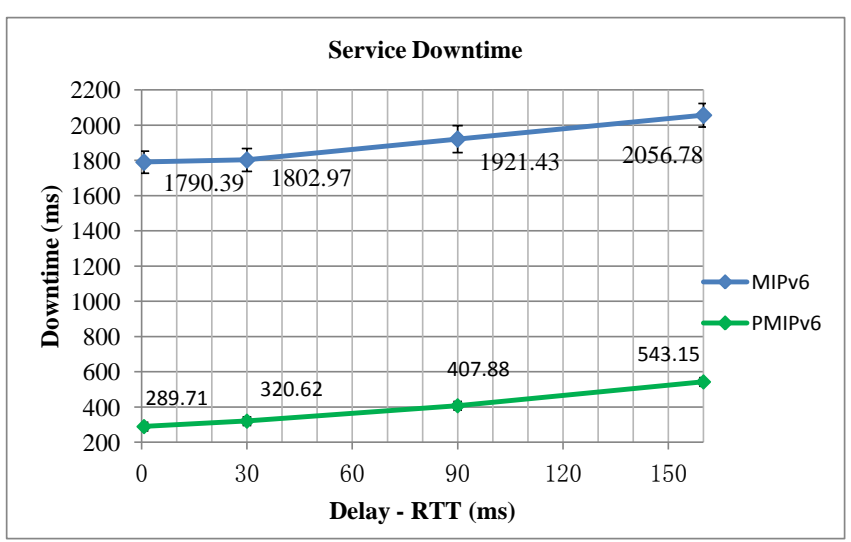

Fig. 5. Average service downtime

TABLE I. PMIPV6 SERVICE DOWNTIME

\begin{tabular}{|c||c|c|c|c|}
\hline \multirow{2}{*}{ Downtime } & \multicolumn{4}{c|}{ Delay - RTT } \\
\cline { 2 - 5 } & $0.02 \mathrm{~ms}$ & $30 \mathrm{~ms}$ & $90 \mathrm{~ms}$ & $160 \mathrm{~ms}$ \\
\cline { 2 - 5 } & Campus & Europe & Trans-Atlantic & Trans-Pacific \\
\hline \hline Average (ms) & 289.71 & 320.62 & 407.88 & 543.15 \\
\hline Minimum(ms) & 254.80 & 275.10 & 351.73 & 440.04 \\
\hline Maximum (ms) & 346.48 & 395.68 & 491.89 & 735.95 \\
\hline Std. dev. (ms) & 8.80 & 22.20 & 30.69 & 55.08 \\
\hline $95 \%$ CI & \pm 4.48 & \pm 5.29 & \pm 7.32 & \pm 13.13 \\
\hline
\end{tabular}

TABLE II. MIPV6 SERVICE DOWNTIME

\begin{tabular}{|c||c|c|c|c|}
\hline \multirow{2}{*}{\multicolumn{1}{|c||}{ Downtime }} & \multicolumn{4}{c|}{ Delay - RTT } \\
\cline { 2 - 5 } & $0.02 \mathrm{~ms}$ & $30 \mathrm{~ms}$ & $90 \mathrm{~ms}$ & $160 \mathrm{~ms}$ \\
\cline { 2 - 5 } & Campus & Europe & Trans-Atlantic & Trans-Pacific \\
\hline \hline Average (ms) & 1790.39 & 1802.97 & 1921.43 & 2056.78 \\
\hline Minimum(ms) & 1294.91 & 1316.05 & 1399.18 & 1583.94 \\
\hline Maximum (ms) & 2576.22 & 2307.80 & 2991.69 & 2664.06 \\
\hline Std. dev. (ms) & 263.02 & 272.34 & 321.13 & 278.00 \\
\hline $95 \%$ CI & \pm 62.71 & \pm 64.94 & \pm 76.57 & \pm 66.76 \\
\hline
\end{tabular}

\section{Performance Results}

We used the following three delay values to emulate WANs with different geographical scope: $30 \mathrm{~ms}$ (Europe), $90 \mathrm{~ms}$ (Trans-Atlantic) and $160 \mathrm{~ms}$ (Trans-Pacific). The chosen values are the average latency guarantees, expressed as round-trip times (RTTs), specified in the SLA for networks operated by Verizon Enterprise Solutions [11]. Normally, when no extra delay is introduced on LMA's links, the average RTT between Client and VM is $0.02 \mathrm{~ms}$.

Where applicable, we have computed the statistical average value over the 70 runs together with associated $95 \%$ confidence intervals (CIs).

\section{A. Service Downtime}

Service downtime or outage is an interruption in service availability during which users are not able to access services running on VM.

To measure service downtime, we developed a simple client-server program that sends a stream of UDP packets from Client to the migrating VM. The stream is started immediately after invoking VM migration via a Secure Socket
Shell (SSH) connection between Client and VM's host. The client side of our program runs on Client and the server side runs on VM.

Our server program acknowledges receipt of client's packets by sending response UDP packets. The client uses an interval of $10 \mathrm{~ms}$ between consecutive UDP packets. Each such packet carries a timestamp, a sequence number and a wellknown string. The timestamp and the sequence number are useful in estimating downtime, total migration time and packet loss. The well-known string eases packet identification when we capture network traffic.

The server reflects back the timestamps in its acknowledgement packets and increases the sequence number by one. Hence, the client side will know when a packet was lost due to downtime (i.e., when VM is paused during final stage of migration). The service downtime is computed by subtracting the timestamp of the packet acknowledged just before the downtime from the timestamp of the first packet received after the VM has resumed operation. The acknowledgment received after downtime marks successful VM migration, at which point the client stops the stream.

Fig. 5 displays the downtime results for PMIPv6 and MIPv6 as a function of different network delays. The same information is shown also in Table I and Table II.

The downtime experienced in PMIPv6 approach is caused by two factors. The first one is related to Xen. During the stop-and-copy phase of memory state transfer, Xen suspends VM and copies the remaining memory pages, the so-called Writable Working Set, which could not be transfered during pre-copy [12]. The second factor is due to the handoff latency in PMIPv6. The duration between detecting VM's attachment and sending to it the RA message containing HNPs represents the downtime component attributed to PMIPv6.

Similarly, the downtime experienced in MIPv6 approach has a component due to Xen, as above, and another due to MIPv6. After VM receives a RA message from the new access router on the foreign link, it configures its CoA with the received prefix and then sends a Binding Update (BU) message to its HA (i.e., to LMA in Fig. 4). The HA updates its routing state and then sends a Binding Acknowledgement (BA) message in reply. First at this point is VM ready to resume communication with Client.

MIPv6 has a considerably larger handoff latency compared to PMIPv6. The main reason for it is the use of the Duplicate Address Detection (DAD) [6] procedure in MIPv6. For PMIPv6, the VM does not perform DAD because the same HNP is advertised in the RA messages and all the MAGs use the same link-local address on the access link shared with VM [13]. However, in the case of MIPv6, the access routers advertise different prefixes in their RA messages and typically use different link-local address on their access links. As a result, VM is forced to perform DAD on both of its link-local and new CoA. DAD causes a minimum delay of one second if the default settings are used. 


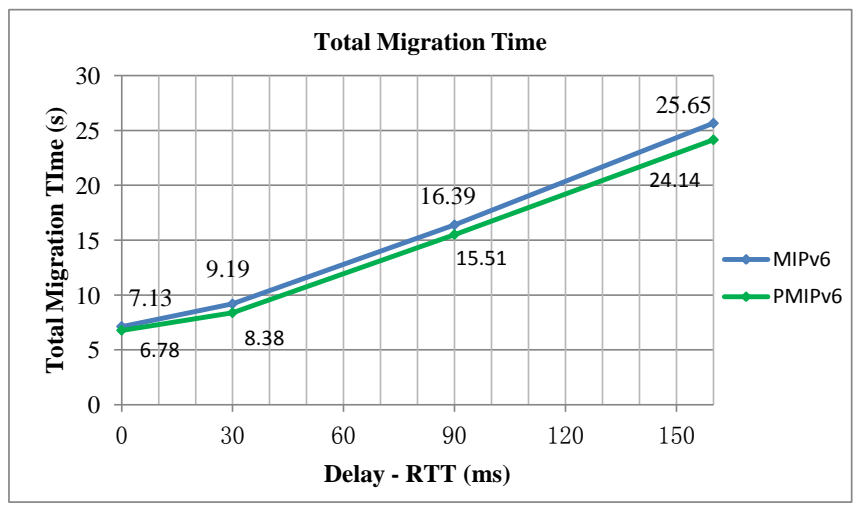

Fig. 6. Average total migration time

TABLE III. PMIPv6 TOTAL MIGRATION TIME

\begin{tabular}{|c||c|c|c|c|}
\hline \multirow{2}{*}{\begin{tabular}{c}
\multirow{2}{*}{$\begin{array}{c}\text { Migration } \\
\text { time }\end{array}$} \\
\cline { 2 - 5 }
\end{tabular}} & $0.02 \mathrm{~ms}$ & $30 \mathrm{~ms}$ & $90 \mathrm{~ms}$ & $160 \mathrm{~ms}$ \\
\cline { 2 - 5 } & Campus & Europe & Trans-Atlantic & Trans-Pacific \\
\hline \hline Average (s) & 6.78 & 8.38 & 15.51 & 24.14 \\
\hline Minimum(s) & 6.00 & 8.00 & 15.00 & 23.05 \\
\hline Maximum (s) & 7.03 & 9.03 & 16.04 & 25.02 \\
\hline Std. dev. (s) & 0.42 & 0.49 & 0.50 & 0.38 \\
\hline $95 \%$ CI & \pm 0.10 & \pm 0.12 & \pm 0.12 & \pm 0.09 \\
\hline
\end{tabular}

TABLE IV. MIPv6 TOTAL MIGRATION TIME

\begin{tabular}{|c||c|c|c|c|}
\hline \multirow{2}{*}{\begin{tabular}{c}
\multirow{2}{*}{$\begin{array}{c}\text { Migration } \\
\text { time }\end{array}$} \\
\cline { 2 - 5 }
\end{tabular}} & $0.02 \mathrm{~ms}$ & $30 \mathrm{~ms}$ & $90 \mathrm{~ms}$ & $160 \mathrm{~ms}$ \\
\cline { 2 - 5 } & Campus & Europe & Trans-Atlantic & Trans-Pacific \\
\hline \hline Average (s) & 7.13 & 9.19 & 16.39 & 25.65 \\
\hline Minimum(s) & 6.25 & 8.42 & 15.62 & 24.76 \\
\hline Maximum (s) & 8.97 & 10.07 & 17.59 & 29.00 \\
\hline Std. dev. (s) & 0.50 & 0.40 & 0.44 & 0.79 \\
\hline $95 \%$ CI & \pm 0.12 & \pm 0.10 & \pm 0.11 & \pm 0.19 \\
\hline
\end{tabular}

\section{B. Total Migration Time}

Total migration time expresses the overall time taken to transfer the entire state ${ }^{1}$ of $\mathrm{VM}$ from one host to the other. This period includes time taken by PMIPv6 (or MIPv6, in case of [5]) to maintain network connectivity. Total migration time is measured using the same UDP tool described above. Total migration time is determined by taking the difference between timestamps of the first and the last packets acknowledged.

Fig. 6 shows the total migration time results obtained when VM was migrated between MAG1 and MAG2. The same information is presented also in Table III and Table IV.

Like downtime, the total migration time has two components. The major component is attributed to Xen, which transfered $256 \mathrm{MB}$ of VM memory state in the iterative pre-copy phase. The second component, PMIPv6 and MIPv6 handoff latencies, constitutes only a small part of the total migration time. The PMIPv6 approach appears to have slightly smaller total migration time compared to the MIPv6 approach. Again, we suspect this is due to DAD being used in MIPv6's case.

\footnotetext{
${ }^{1}$ Disk state is excluded since a shared storage medium is used during our experiments.
}

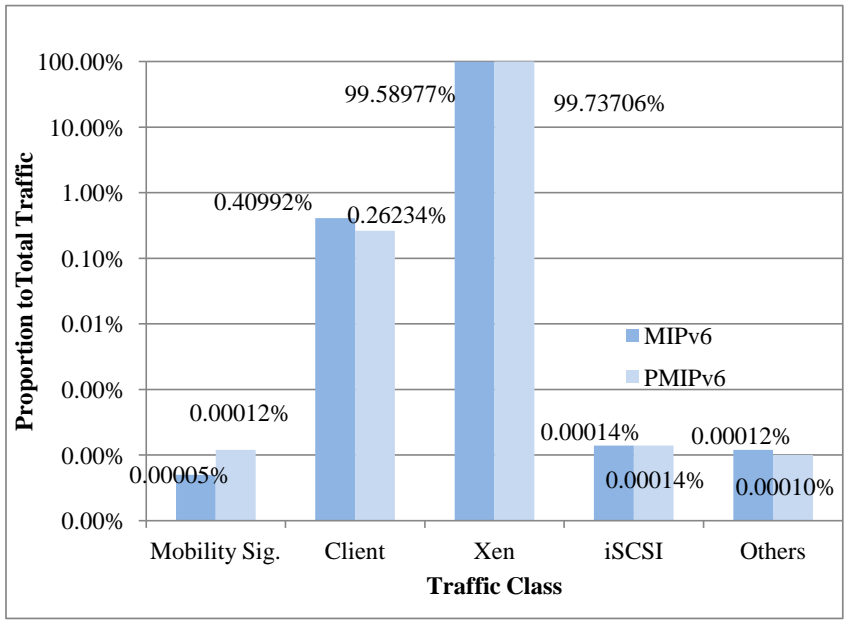

Fig. 7. Signaling overhead

\section{Survivability of TCP Sessions}

This metric expresses the likelihood that a TCP session established between the user Client and the migrating VM can be preserved during and after migration.

We developed another client-server program to test this. The client side runs on VM and the server side runs on Client. The server creates a TCP socket and listens for incoming connection requests. The client, after connecting to the server, sends TCP data repeatedly at an interval of $10 \mathrm{~ms}$. The Linux $r e c v()$ system call is used to receive the client's messages on the server. The call blocks until data is available or an error has occurred. If the call is successful, it returns the length of the received message. A return value of -1 indicates failure while a return value of 0 indicates that the client has closed the TCP connection. Therefore, -1 or 0 return values indicate that the TCP session was lost. Otherwise, the TCP session is considered to have survived after VM's migration.

The TCP session survived the migration in all 70 runs executed using the PMIPv6 and MIPv6 approach, respectively. Therefore, we can conclude that both solutions are transparent to the transport layer in the migrating VM.

\section{Signaling Overhead}

Using tshark ${ }^{2}$, we captured all the traffic crossing LMA's eth0 and eth1 interfaces that connect LMA to MAG1 and MAG2, respectively. The captured traffic was processed offline and the packets were classified into five categories: Xen traffic, client traffic, mobility signaling, iSCSI traffic and others.

Fig. 7 illustrates the average proportion of the total traffic volume of each of these traffic categories computed over 10 experiment repetitions. On average, the total traffic volume that crossed LMA's eth 0 and ethl interfaces during a single VM migration session is $541.86 \mathrm{MB}$ for the PMIPv6 approach and 545.55 MB for the MIPv6 approach. Note that data transfered

${ }^{2}$ Tshark is terminal-oriented version of wireshark that can be used to capture packet from live network and read packets from previously saved captured files. Its native capture file format is libcap format, the same format used by tcpdump and various others. 
between MAG1 and MAG2 is accounted for twice since we captured traffic on the two LMA interfaces connected to them.

Traffic crossing LMA's interfaces is almost entirely related to Xen. Xen contributed with more than $99 \%$ of the total traffic that crossed the LMA's links, irrespective if MIPv6 or PMIPv6 is used. The traffic is generated by VM's $256 \mathrm{MB}$ memory state that needed to be copied from the source host to the destination.

Client, iSCSI, and other traffic categories accounted for less than $0.05 \%$ of the total. iSCSI traffic includes packets exchanged between MAG1, MAG2 and isCSI-Server. In our experiments, VM did not perform any activity that required disk access. This explains the small iSCSI traffic volume, only $0.00014 \%$ of the total traffic.

Mobility related signaling traffic includes packets related to AAA, BU, BA, PBU and PBA messages. Packets related to IPv6 Neighbor Discovery and IPv6 Stateless Address Autoconfiguration are also included in this category. This type of traffic represents a negligible part of the total traffic, less than $0.00012 \%$ in both approaches. Thus, the network overhead caused by mobility related signals can be considered insignificant compared to Xen's live migration traffic.

\section{CONCLUDING REMARKS}

According to our results, the downtime experienced with the PMIPv6 approach is considerably shorter than the one generated by the MIPv6 approach.

The PMIPv6 approach may cause inefficient routing because traffic to and from the VM has to go through the LMA. In contrast, MIPv6 using route optimization overcomes this limitation.

Also, there is overhead related to setting up and maintaining tunnels between LMAs and MAGs in the case of PMIPv6, and between MN and HA in the case of MIPv6. However, a PMIPv6 tunnel can be used simultaneously by multiple VMs hosted on the same node or on a set of nodes (e.g., a data center). In this respect, the PMIPv6 approach is more efficient than the MIPv6 approach, where a tunnel has to be established per VM.

Research is being carried out to provide inter-domain mobility support for PMIPv6. In addition, IETF's NetworkBased Mobility Extensions (netext) Working Group is working on extending PMIPv6 specification to support enhanced multihoming, intertechnology handoff and route optimization. In our opinion, these are strong arguments in favor of the PMIPv6 approach to live migration.

\section{REFERENCES}

[1] H. Soliman, Mobile IPv6: Mobility in a Wireless Internet. Boston, MA, USA: Addison Wesley, 2004, ISBN: 0-201-78897-7.

[2] T. Wood, P. Shenoy, K. Ramakrishnan, and J. Van Der Merwe, "CloudNet: Dynamic pooling of cloud resources by live WAN migration of virtual machines," in Proceedings of VEE 2011, Newport Beach, CA, USA, Mar. 2011

[3] S. Gundavelli, K. Leung, V. Devarapalli, K. Chowdhury, and B. Patil, RFC 5213: Proxy Mobile IPv6, IETF, Aug. 2008.

[4] R. Wakikawa and S. Gundavelli, RFC 5844: IPv4 Support for Proxy Mobile IPv6, IETF, May 2010. [Online]. Available: http: //tools.ietf.org/html/rfc5844
[5] M. M. Harney, Goasguen and Westall, "The efficacy of live virtual machine migrations over the Internet," in Proceedings of VTDC'07, Reno, NV, United states, Nov. 2007.

[6] T. Narten, W. A. Simpson, E. Nordmark, and H. Soliman, RFC 4861: Neighbor Discovery for IP version 6 (IPv6), IETF, Sep. 2007. [Online]. Available: http://tools.ietf.org/html//rfc4861

[7] Open air interface proxy mobile IPv6 (OAI PMIPv6). EURECOM. Retrieved: Feb., 2014. [Online]. Available: http://www.openairinterface. org/openairinterface-proxy-mobile-ipv6-oai-pmipv6

[8] UMIP - mobile IPv6 and NEMO basic support implementation for linux. Retrieved: Feb., 2014. [Online]. Available: http://umip.org/

[9] Linux IPv6 router advertisement daemon (radvd). Retrieved: Feb., 2014. [Online]. Available: http://www.litech.org/radvd/

[10] C. E. Perkins, D. B. Johnson, and J. Arkko, RFC 6275: Mobility Support in IPv6, IETF, Jul. 2011. [Online]. Available: http://tools.ietf.org/html/rfc6275

[11] IP latency statistics - Verizon enterprise solutions. Retrieved: Sep., 2013. [Online]. Available: http://www.verizonenterprise.com/about/ network/latency

[12] H. Liu, H. Jin, C.-Z. Xu, and X. Liao, "Performance and energy modeling for live migration of virtual machines," Cluster Computing, vol. 16, no. 2, pp. 249-264, Jun. 2011.

[13] K.-S. Kong, W. Lee, Y.-H. Han, M.-K. Shin, H.-R. You, and W. Lee, "Mobility management for all-IP mobile networks: Mobile IPv6 vs. proxy mobile IPv6," IEEE Wireless Commun. Mag., vol. 15, no. 2, pp. 36-45, Apr. 2008. 